OPEN ACCESS

Edited by:

Hubert Preissl,

Institute for Diabetes Research and

Metabolic Diseases (IDM), Germany

Reviewed by:

Onno Meijer,

Leiden University, Netherlands Jorge Miguel Amaya Fernandez, Leiden University, Netherlands, in collaboration with reviewer OM

Erwin Lemche, King's College London,

United Kingdom

*Correspondence:

Cong Zhou

doctorzhoucong@163.com

Specialty section:

This article was submitted to Neuroendocrine Science,

a section of the journal

Frontiers in Endocrinology

Received: 25 January 2021 Accepted: 07 April 2021 Published: 03 May 2021

Citation:

Zhou C, Li J, Dong M, Ping L, Lin H,

Wang $Y$, Wang $S$, Gao $S, Y u$ G,

Cheng $Y$ and $X u X$ (2021) Altered White Matter Microstructures in Type 2

Diabetes Mellitus: A Coordinate-

Based Meta-Analysis of Diffusion

Tensor Imaging Studies.

Front. Endocrinol. 12:658198.

doi: 10.3389/fendo.2021.658198

\section{Altered White Matter Microstructures in Type 2 Diabetes Mellitus: A Coordinate-Based Meta-Analysis of Diffusion Tensor Imaging Studies}

\author{
Cong Zhou ${ }^{1 *}$, Jie $\mathrm{Li}^{2}$, Man Dong ${ }^{1}$, Liangliang Ping ${ }^{3}$, Hao Lin ${ }^{1}$, Yuxin Wang ${ }^{1}$, \\ Shuting Wang ${ }^{1}$, Shuo Gao ${ }^{1}$, Ge Yu ${ }^{1}$, Yuqi Cheng ${ }^{4}$ and Xiufeng $X u^{4}$ \\ 1 School of Mental Health, Jining Medical University, Jining, China, ${ }^{2}$ Department of Psychiatry, Jining Psychiatric Hospital, \\ Jining, China, ${ }^{3}$ Department of Psychiatry, Xiamen Xianyue Hospital, Xiamen, China, ${ }^{4}$ Department of Psychiatry, The First \\ Affiliated Hospital of Kunming Medical University, Kunming, China
}

Objective: Type 2 diabetes mellitus (T2DM) is often accompanied by cognitive decline and depressive symptoms. Numerous diffusion tensor imaging (DTI) studies revealed microstructural white matter (WM) abnormalities in T2DM but the findings were inconsistent. The present study aimed to conduct a coordinate-based meta-analysis (CBMA) to identify statistical consensus of DTI studies in T2DM.

Methods: We performed a systematic search on relevant studies that reported fractional anisotropy (FA) differences between T2DM patients and healthy controls ( $\mathrm{HC}$ ). The anisotropic effect size seed-based d mapping (AES-SDM) approach was used to explore WM alterations in T2DM. A meta-regression was then used to analyze potential influences of sample characteristics on regional FA changes.

Results: A total of eight studies that comprised 245 patients and $200 \mathrm{HC}$, along with 52 coordinates were extracted. The meta-analysis identified FA reductions in three clusters including the left inferior network, the corpus callosum (CC), and the left olfactory cortex. Besides, FA in the CC was negatively correlated with body mass index (BMI) in the patients group.

Conclusions: T2DM could lead to subtle WM microstructural alterations, which might be associated with cognitive deficits or emotional distress symptoms. This provides a better understanding of the pathophysiology of neurodegeneration and complications in T2DM.

Systematic Review Registration: Registered at PROSPERO (http://www.crd.york.ac. uk/PROSPERO), registration number: CRD42020218737.

Keywords: type 2 diabetes mellitus, diffusion tensor imaging, fractional anisotropy, white matter, meta-analysis 


\section{INTRODUCTION}

The International Diabetes Federation estimates that 415 million people have diabetes mellitus worldwide, with $90 \%$ of these individuals having T2DM (1). Type 2 diabetes mellitus (T2DM) is a chronic metabolic disorder characterized by reduced insulin sensitivity, followed by a compensatory increase in insulin secretion (2). The disease has become a critical health concern worldwide owing to its high prevalence and related disability and mortality (3). T2DM usually leads to various complications in multiple organs, including impairments in the brain (4). People with type 2 diabetes are at an increased risk of cognitive decline and dementia (including Alzheimer's disease, AD) $(5,6)$, which is related with worse diabetes management, more frequent occurrence of severe hypoglycemic episodes, and an increased risk of cardiovascular events, and death (7). Earlier meta-analyses showed that the presence of diabetes in older adults was associated with $47 \%$ increased risk of all dementia, 39\% increased risk of AD, and $138 \%$ increased risk of vascular dementia $(8,9)$. Existing evidence indicated that microstructural brain atrophy contributed to poor cognitive function (10-13). Several neuroimaging studies with different modalities have demonstrated that T2DM is accompanied with structural and functional abnormalities in various regions of the brain $(2,14,15)$. Moreover, T2DM and mood disorders share pathophysiological commonalities in the central nervous system $(16,17)$. The prevalence of depression among T2DM is quite high (18-21), which is considered to be related with cerebral microvascular dysfunction (22). However, the specific neurobiological mechanisms underlying the cognitive impairment and emotional distress of T2DM patients remain unclear for now.

Advances in MRI techniques make it possible to investigate subtle structural alterations of the brain. Among them, diffusion tensor imaging (DTI) is able to detect white matter (WM) microstructure characteristics by estimating random movement of water molecules in the brain (23). The most widely used parameter to study DTI is fractional anisotropy (FA), which reflects diffusion direction and is related to fiber orientation. Any reduction in white matter anisotropy indicates an alteration in the degree of tissue order or integrity (24). DTI approach is widely applied in the evaluation of WM microstructure in various central nervous system disorders. Specially, DTI metrics appears to be a more sensitive marker of cognitive decline due to aging and $\mathrm{AD}$, even when there is no sign of microstructural gray matter (GM) volume alterations and atrophy of brain structures $(25,26)$. The two most widely used methods of DTI to achieve whole-brain analysis were voxelbased analysis (VBA) and tract-based spatial statistics (TBSS) (27). The former involves analyzing all white matter voxels and correcting for multiple comparisons and noise by reporting only contiguous clusters of significant voxels, while the latter isolates the central core of white matter tracts with the highest FA and reports significant clusters within that white matter skeleton (28, 29). Findings from numerous studies have suggested widespread white matter abnormalities in T2DM patients. However, the results are inconsistent and controversial. According to previous studies, significantly decreased in FA has been observed in patients with T2DM in widespread WM regions such as the frontal lobe $(15,30,31)$, temporal region $(15,30-33)$, corpus callosum (CC) (34-36), cingulum bundle $(15,35,37)$, uncinate fasciculus (UF) $(35,36,38)$, and corticospinal tract (CST) $(35$, 36). The inconsistencies of different studies were probably owing to small sample size, heterogeneous demographic characteristics of the patients, and the diversity of methodological techniques.

The coordinate-based meta-analysis (CBMA) is a widely used method to solve the discrepancies of regional alterations among various neuroimaging studies (39). The anisotropic effect size seed-based d mapping (AES-SDM) is an advanced statistical technique for CBMA on different neuroimaging techniques such as structural MRI, functional MRI, DTI, or PET (40). Compared with earlier methods such as activation likelihood estimation and multilevel kernel density analysis $(41,42)$, the AES-SDM has strengths as below: (a) In the AES-SDM, both positive and negative differences in the same map are combined to avoid a particular voxel from appearing to be significant in opposite directions (43); (b) The AES-SDM approach allows reported peak coordinates to be combined with statistical parametric maps, thus ensuring more exhaustive and accurate metaanalyses (44); (c) SDM enables several complementary analyses, such as jack-knife, subgroup, and meta-regression analyses, which can be used to evaluate the robustness and heterogeneity of the results (40). The AES-SDM method has been fully validated in several neuropsychiatric disorders including Parkinson's disease $(45,46)$, major depressive disorder (MDD) (29), bipolar disorder (47), obsessive-compulsive disorder $(43,48,49)$, autism spectrum disorder (50), type 1 diabetes mellitus (T1DM) (51), and also in voxel-based morphometry (VBM) studies in T2DM patients $(52,53)$.

A recently published systematic review of DTI studies (54) comprehensively and systematically summarized previous DTI findings of brain microstructural abnormalities in T2DM. However, this review study is not able to detect the discrepancies of regional alterations with reported coordinates and anisotropic effect size. Thus, a CBMA using AES-SDM is required to identify consistent results from DTI studies in patients with T2DM. The first objective of this present research was to investigate the most robust FA alterations in T2DM compared with healthy controls (HC). Secondly, we intended to explore the potential effects of demographics and clinical characteristics including mean age, duration of disease, body mass index (BMI), and $\mathrm{HbAlc} \%$ on WM changes by using meta-regression approach. We hypothesized that patients with T2DM would exhibit microarchitecture alterations in core WM tracts such as the CC, as well as regions related with cognitive functions and emotional regulations.

\section{MATERIALS AND METHODS}

\section{Literature Search Strategy}

This meta-analysis was conducted according to the Preferred Reporting Items for Systematic Reviews and Meta-Analyses (PRISMA) guidelines (55-57). The protocol of this CBMA was 
registered at PROSPERO (http://www.crd.york.ac.uk/ PROSPERO) (registration number: CRD42020218737). Systematic and comprehensive searches were used to acquire relevant literatures from the PubMed and Web of Science databases published (or "in press") up to October 31, 2020. The search keywords were ("type 2 diabetes mellitus" or "T2DM" or "type 2 diabetes") and ("diffusion tensor" or "DTI" or "diffusion magnetic resonance imaging"). Additionally, the reference lists of identified studies and relevant reviews were manually checked to avoid omitting.

\section{Study Selection}

Studies which met the following criteria were included (1): studies compared FA value differences between T2DM and $\mathrm{HC}$ in whole-brain analyses (2); reported results in Talairach or Montreal Neurological Institute (MNI) coordinates; (3) used a threshold for significance; (4) articles written in the English language and published in peer-reviewed journals. Exclusion criteria were: (1) meta-analysis, reviews, case reports, or tractography-based only study; (2) studies with no direct between-group comparison; (3) studies from which peak coordinates or parametric maps were unavailable.

\section{Quality Assessment and Data Extraction}

Two authors (ZC and LJ) independently searched the literatures, assessed the quality of the retrieved articles, extracted and crosschecked the data from eligible articles. The quality of the final studies was also independently checked by both authors following guidelines for neuroimaging meta-analyses promoted by Müller and colleagues (58). For each study the following data were recorded: first author, cohort size, demographics (age and gender), illness duration, BMI, HbAlc\%, imaging parameters, data processing method and statistical threshold, as well as the three-dimensional peak coordinates of case-control differences in each study.

\section{AES-SDM Meta-Analysis}

Regional FA differences between T2DM patients and HC were performed using the SDM software v5.15 (http://www. sdmproject.com) $(43,59)$ in a voxel-based meta-analysis approach. We conducted the analysis according to the SDM tutorial and previous meta-analytic studies. The AES-SDM technique uses effect sizes combining with reported peak coordinates which are extracted from databases with statistical parametric maps, and recreates maps of the original maps of the effect size of FA between patients and controls, rather than just assessing the probability or likelihood of a peak (40).

The AES-SDM procedures have been described in detail elsewhere $(29,46,60)$, and were briefly summarized as follows: (1) The peak coordinates of all white-matter from each data set were extracted at the level of $t$-statistics $(Z$ - or $P$ - values for significant clusters which were then converted to $t$-statistics using the SDM online converter); (2) The peak coordinates for each study were recreated using a standard MNI map of the effect size of the group differences in FA by means of an anisotropic Gaussian kernel (44). A relatively wide full width at half maximum $(20 \mathrm{~mm})$ and DTI templates were used to control false-positive results; (3) The standard meta-analysis was conducted to create a mean map via voxel-wise calculation of the random-effects mean of the study maps. According to Radua et al. (40), an uncorrected $P=0.005$ using the AES-SDM software is approximately equivalent to a corrected $P=0.025$. Here, we used more stringent thresholds as follows: uncorrected $P$ value $<0.001$, peak height threshold $Z=1.00$, and cluster size threshold = 10 voxels.

\section{Sensitivity Analyses}

To assess the replicability of the results, we performed a systematic whole-brain voxel-based jackknife sensitivity analysis. This procedure involved repeating the main statistical analysis for each result eight times, discarding a different study each time. If a brain region remains significant after running jackknife sensitivity in all or most of the combinations of studies, the finding is considered highly replicable (43).

\section{Meta-Regression Analysis}

Considering the potential influences of mean age, duration of disease, BMI, and $\mathrm{HbAlc} \%$ on WM abnormalities, a more conservative threshold $(P<0.0005)$ was adopted in consistent with previous meta-analyses and the recommendations of the AES-SDM authors (43), and only brain regions identified in the main effect were considered.

\section{RESULTS}

\section{Included Studies and Sample Characteristics}

The flow diagram of the identification and the attributes of the studies is presented in Figure 1. The demographics of the samples are summarized in Table 1. The search strategy identified 90 studies, eight of which met the inclusion criteria $(15,30,32,34-36,61,62)$. One study contained two different subgroups of T2DM patients (T2DM patients with mild cognitive impairment and T2DM patients with normal cognition), but only the coordinates of significantly different clusters in T2DM patients with mild cognitive impairment were reported. We treated this study as one single dataset. Thus, our final sample comprised 245 T2DM patients and $200 \mathrm{HC}$, along with 52 coordinates extracted from eight datasets. The scanning methods and FA alterations of the eight datasets are shown in Table 2.

\section{Regional Differences in FA}

The meta-analysis revealed that patients with T2DM exhibited significant FA reductions in three clusters relative to $\mathrm{HC}$, including the left inferior network, the CC and left olfactory cortex (BA 25), as illustrated in Figure 2 and Table 3. No region with higher FA was identified in the current meta-analysis.

\section{Jackknife Sensitivity Analysis}

The whole-brain jackknife sensitivity analysis revealed that decreased FA in T2DM patients in the left inferior network 

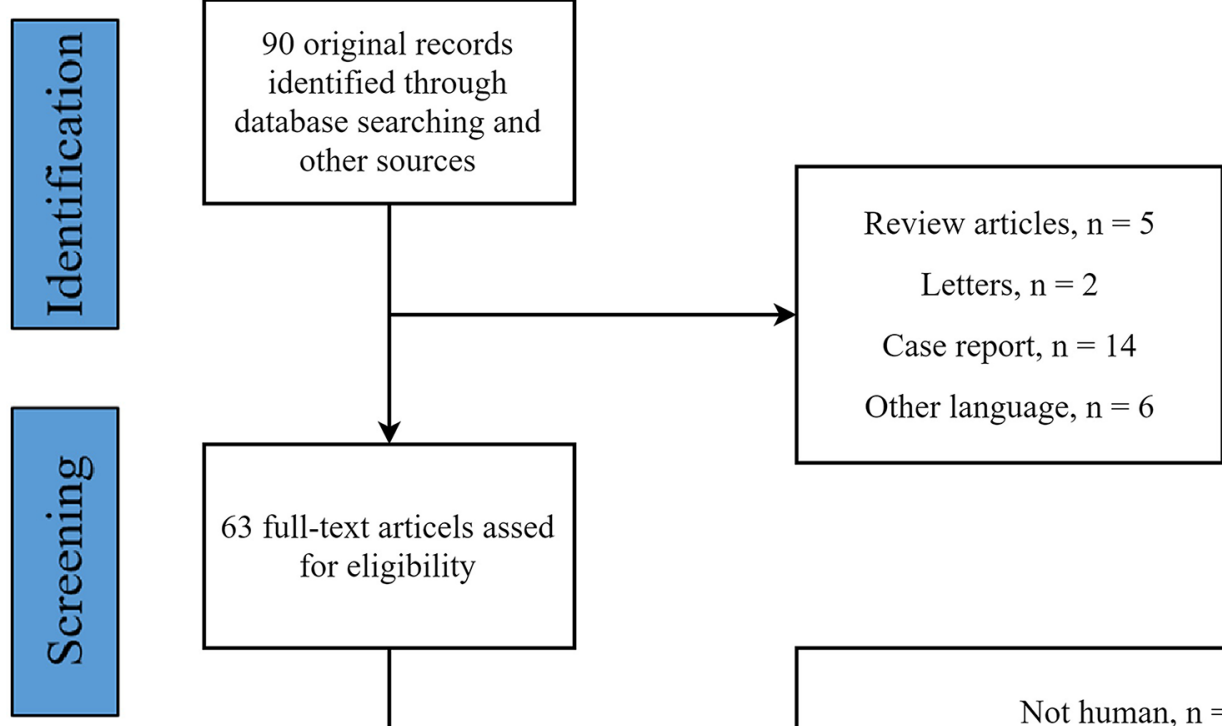

63 full-text articels assed for eligibility
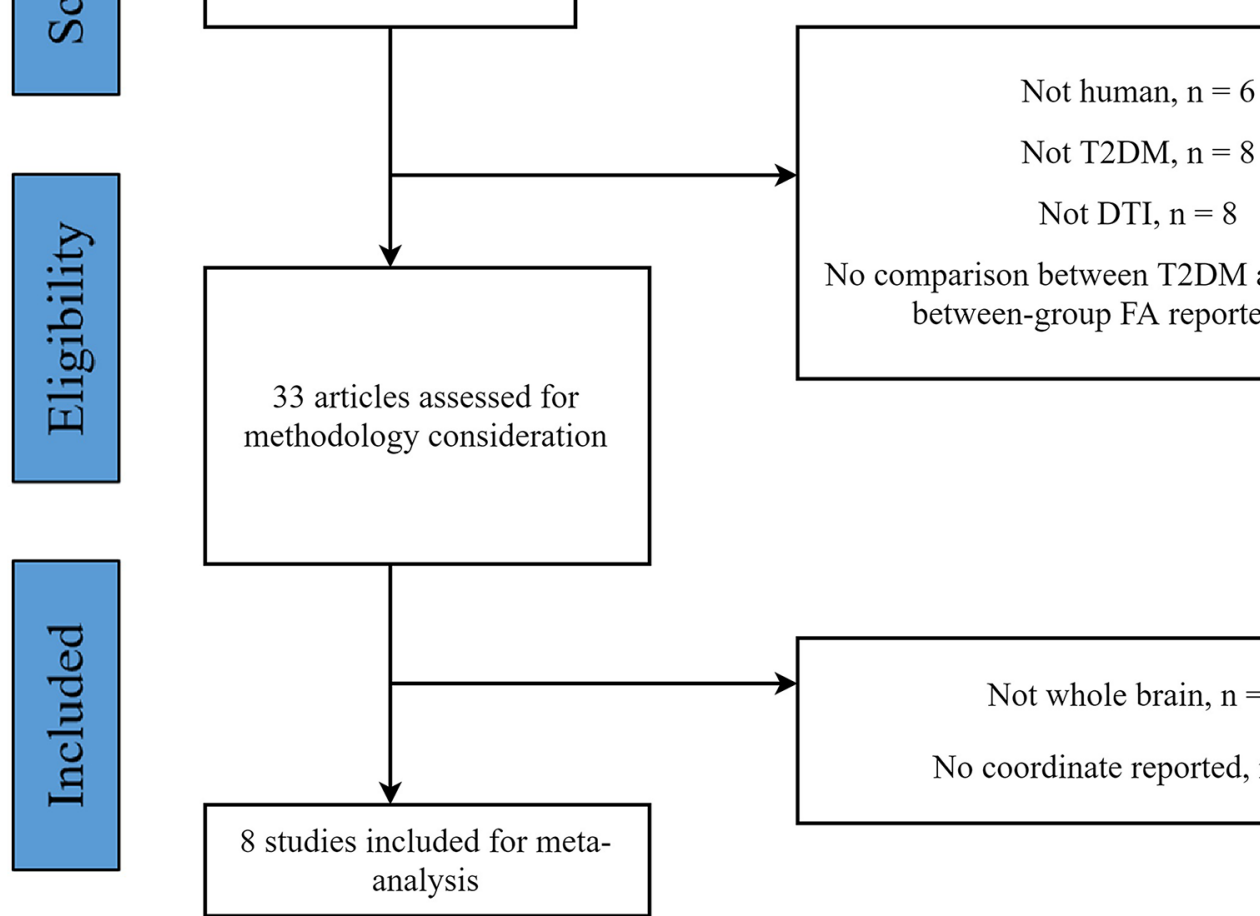

No comparison between T2DM and HC, or No between-group FA reported, $\mathrm{n}=8$

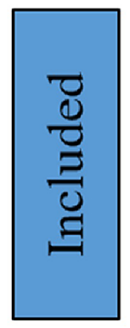

Not whole brain, $\mathrm{n}=9$

No coordinate reported, $\mathrm{n}=16$

FIGURE 1 | Flow diagram for the identification and exclusion of studies.

and the CC was highly replicable, as these findings were preserved throughout all but one combination of the datasets. FA reduction in the left olfactory cortex remained significant in all but two combinations (Table 3 ).

\section{Meta-Regression Analysis}

At a stringent threshold of $P<0.0005$, meta-regression analysis found a negative correlation between FA in the CC and BMI in the patients group (Table 4). The mean age of patients, illness duration, and $\mathrm{HbAlc} \%$ were not linearly associated with FA changes.

\section{DISCUSSION}

To our knowledge, this study is the first coordinate-based metaanalysis (CBMA) of DTI studies in T2DM patients investigating microstructural WM abnormalities and examining how clinical features affect WM morphometry. Using the AES-SDM metaanalytical approach, this study identified decreased FA in three clusters, and these three regional differences remained replicable in the Jackknife sensitivity analyses. The largest cluster exhibited a peak coordinate in the left inferior network mainly consisted of left inferior fronto-occipital fasciculus (IFOF), left inferior longitudinal 
TABLE 1 | Demographic and clinical characteristics of the participants in eight studies included in the meta-analysis.

\begin{tabular}{|c|c|c|c|c|c|c|c|c|}
\hline \multirow[t]{2}{*}{ Study } & \multicolumn{2}{|c|}{ Subjects, $\mathrm{n}$ (female, $n$ ) } & \multicolumn{2}{|c|}{ Age, years } & \multirow[t]{2}{*}{ Diabetes duration, years } & \multirow[t]{2}{*}{ HbAlc $\%$} & \multirow[t]{2}{*}{ BMIkg/m² } & \multirow[t]{2}{*}{ Comorbidity (number of patients) } \\
\hline & T2DM & HC & T2DM & HC & & & & \\
\hline Yau et al. (30) & $24(11)$ & $17(9)$ & 57.2 & 56.4 & 7.9 & 7.8 & 32.1 & Hypertension (16) \\
\hline Yau et al. (15) & $18(\mathrm{~N} / \mathrm{A})$ & $18(\mathrm{~N} / \mathrm{A})$ & 16.5 & 17.2 & 2.6 & 8.3 & 37.7 & $\begin{array}{l}\text { Obesity (18) } \\
\text { Hypertension (5) }\end{array}$ \\
\hline Kim et al. (34) & $20(11)$ & $20(11)$ & 54.6 & 54.3 & 12.1 & 10.7 & 24.7 & $\begin{array}{l}\text { Diabetic retinopathy (9) } \\
\text { Diabetic nephropathy (4) } \\
\text { Diabetic peripheral nephropathy (7) }\end{array}$ \\
\hline van Bloemendaal et al. (61) & $16(8)$ & $15(7)$ & 61.4 & 57.3 & 7.0 & 6.9 & 34.0 & Obesity (16) \\
\hline Nouwen et al. (35) & $13(13)$ & $20(14)$ & 16.0 & 16.1 & 2.6 & 7.8 & $\mathrm{~N} / \mathrm{A}$ & $\mathrm{N} / \mathrm{A}$ \\
\hline Yoon et al. (36) & $100(50)$ & $50(25)$ & 49.2 & 49.0 & 1.8 & 7.1 & 25.5 & Overweight/obesity (50) \\
\hline Liang et al. (62) & $34(24)$ & $32(14)$ & 58.3 & 56.3 & 6.9 & 7.9 & 24.4 & $\begin{array}{l}\text { Overweight (20) } \\
\text { Obesity (1) } \\
\text { Hypertension (9) }\end{array}$ \\
\hline Xiong et al. (32) & $20(12)$ & $28(18)$ & 63.6 & 59.7 & 9.1 & 8.2 & 24.4 & Mild cognitive impairment (20) \\
\hline
\end{tabular}

T2DM, type 2 diabetes mellitus; HC, healthy controls; N/A, not available; BMI, body mass index.

TABLE 2 | Scanning methods and FA alterations of the eight studies included in this meta-analysis.

\begin{tabular}{|c|c|c|c|c|c|c|}
\hline Study & Scanner & $\begin{array}{l}\text { Diffusion } \\
\text { encoding } \\
\text { directions }\end{array}$ & $\begin{array}{l}\text { Type of } \\
\text { analysis }\end{array}$ & Statistical threshold & $\begin{array}{l}\text { Number of } \\
\text { coordinates }\end{array}$ & FA alterations \\
\hline Yau et al. (30) & $1.5 \mathrm{~T}$ & 6 & VBA & $P<0.005$, uncorrected & 6 & $\begin{array}{l}\text { Decrease observed in } L \text { temporal stem, } R \text { prefrontal region, } L \text { frontal } \\
\text { temporal region, } R \text { external capsule, } L \text { parietal region, and } L \text { middle } \\
\text { temporal region }\end{array}$ \\
\hline Yau et al. (15) & $1.5 \mathrm{~T}$ & 6 & VBA & $P<0.005$, uncorrected & 3 & $\begin{array}{l}\text { Decrease observed in R cingulate WM, } L \text { cerebral peduncle, and } L \\
\text { temporal stem }\end{array}$ \\
\hline Kim et al. (34) & $3.0 \mathrm{~T}$ & 30 & TBSS & $P<0.05$, FWE corrected & 10 & $\begin{array}{l}\text { Decrease observed in bilateral posterior thalamic radiation, } \mathrm{R} \\
\text { retrolenticular part of internal capsule, } \mathrm{R} \text { splenium of CC, R fornix } \\
\text { (cres)/stria terminalis, } \mathrm{R} \text { sagittal stratum, R external capsule }\end{array}$ \\
\hline $\begin{array}{l}\text { van Bloemendaal } \\
\text { et al. (61) }\end{array}$ & $3.0 \mathrm{~T}$ & 30 & TBSS & $P<0.05$, FWE corrected & 0 & - \\
\hline Nouwen et al. (35) & $3.0 \mathrm{~T}$ & 61 & TBSS & $P<0.05$, TFCE corrected & 9 & $\begin{array}{l}\text { Decrease observed in } L \text { CST, medial corpus callosum, } L \text { fornix, } L \\
\text { thalamic radiation, } L \text { retrolenticular internal capsule, } L \text { IFOF, } R \text { anterior } \\
\text { corona radiata, the genu of CC, } L \text { uncinate, } L \text { callosal body and } \\
\text { cingulum, } L \text { anterior external capsule, and uncinate fasciculus }\end{array}$ \\
\hline Yoon et al. (36) & $1.5 \mathrm{~T}$ & N/A & VBA & $P<0.05$, corrected & 22 & $\begin{array}{l}\text { Decrease observed in L fornix sagittal stratum, L IFOF, L uncinate } \\
\text { fasciculus, bilateral CST, CC, bilateral anterior thalamic radiation } \\
\text { fornix, R superior corona radiata, bilateral cerebellar WM, bilateral } \\
\text { forceps minor, bilateral optic radiation, bilateral anterior corona } \\
\text { radiata, L external capsule, R parietal WM, and R temporal WM }\end{array}$ \\
\hline Liang et al. (62) & $3.0 \mathrm{~T}$ & 25 & VBA & $\begin{array}{l}P<0.05, \text { AlphaSim } \\
\text { corrected }\end{array}$ & 1 & L corona \\
\hline Xiong et al. (32) & $3.0 \mathrm{~T}$ & 25 & TBSS & $P<0.05$, FWE corrected & 1 & R temporal lobe \\
\hline
\end{tabular}

CC, corpus callosum; CST, corticospinal tract; FA, fractional anisotropy; FWE, family-wise error; IFOF, inferior fronto-occipital fasciculus; N/A, not available; L, left; R, right; T, Tesla; TBSS, tract-based spatial statistics; TFCE, threshold-free cluster enhancement; VBA, voxel-based analysis; WM, white matter.

fasciculus (ILF), left uncinate fasciculus (UF), and anterior commissure. Other clusters exhibited FA reductions in the CC and the left olfactory cortex (BA25). Besides, according to the metaregression, FA in the $\mathrm{CC}$ was negatively correlated with $\mathrm{BMI}$ in the patients group. These findings enhanced our understanding of the underlying neurodegeneration in T2DM.

Our meta-analysis only identified lower FA rather than higher FA in T2DM patients. This is in accordance with most published DTI studies of T2DM (54). As FA presents the anisotropic diffusion of water molecules and can reflect the underlying characteristics of microstructure, such as fiber density, axonal diameter, thickness of the myelin sheaths, and directionality of the fibers $(27,63)$, decreased FA in our findings represented disrupted WM microarchitecture in the brain. One of the core characteristics of T2DM is insulin resistance, which interferes with glucose metabolism and even can lead to increased plasma glucose in regional brain areas in T2DM patients (52). From the microscopic point of view, hyperglycemia is considered to be related with various metabolic and molecular alterations and could result in brain cell dysfunction, degeneration, or death ultimately $(52,64)$. And from the macroscopic perspective, brain atrophy might be the neurobiological basis of cognitive decline $(5,6,11)$. This was also in agreement with previous VBM metaanalyses of T2DM $(52,53)$.

The left inferior network mainly comprised the left IFOF, left ILF, left UF, and anterior commissure. Several studies support the extension of WM impairments in T2DM to other association fibers, 


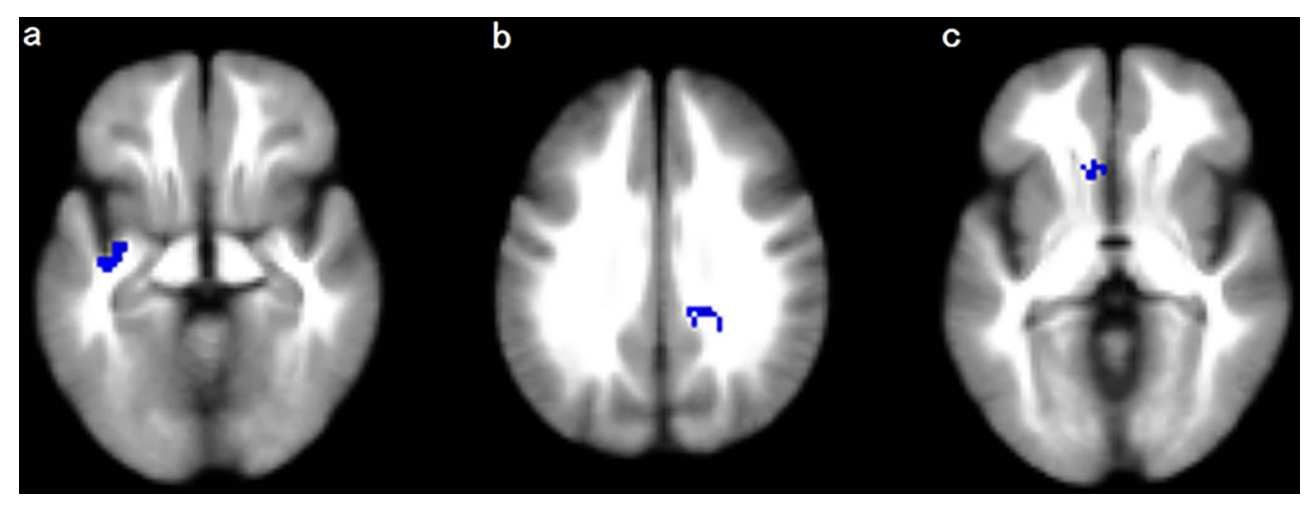

FIGURE 2 | Regions showing FA reductions in (A) the left inferior network; (B) the corpus callosum; and (C) the left olfactory cortex. Significant clusters are overlaid on MRIcron template for Windows for display purposes only.

TABLE 3 | White Matter Regions of FA reductions in T2DM Patients compared to healthy controls in the coordinate-based meta-analysis.

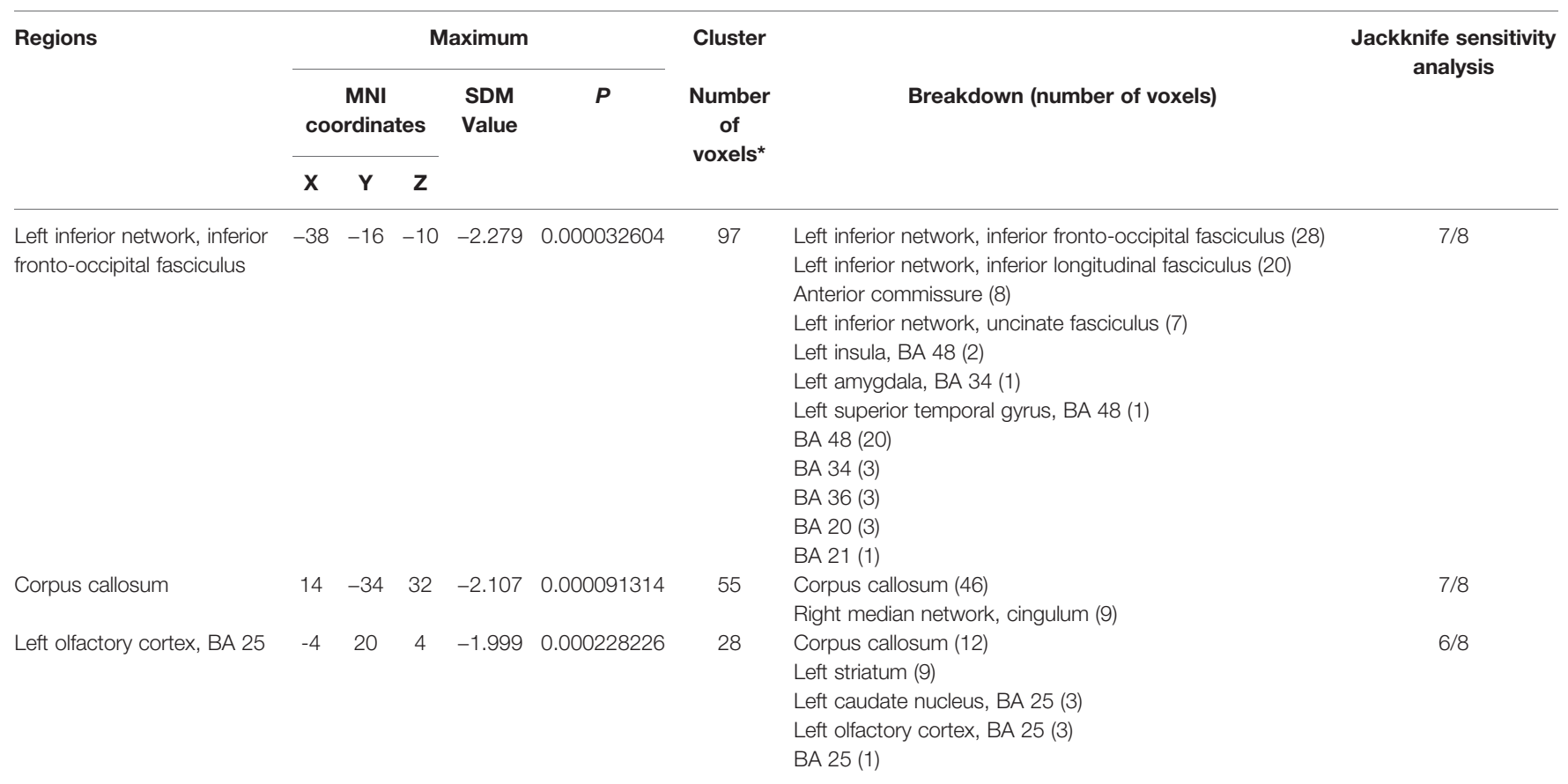

${ }^{*}$ All voxels with $P<0.001$ uncorrected.

BA, Brodmann area; FA, fractional anisotropy; MNI, Montreal Neurological Institute; SDM, seed-based d mapping; T2DM, type 2 diabetes.

TABLE 4 | Correlation between FA alterations and BMI in T2DM revealed by Meta-regression analyses.

\begin{tabular}{|c|c|c|c|c|c|c|c|}
\hline \multirow[t]{2}{*}{ Factor } & \multirow[t]{2}{*}{ Anatomic label } & \multicolumn{3}{|c|}{ MNI coordinates } & \multirow[t]{2}{*}{ SDM Value } & \multirow[t]{2}{*}{$\boldsymbol{P}$} & \multirow[t]{2}{*}{ Number of voxels } \\
\hline & & $\mathbf{x}$ & $\mathbf{Y}$ & $\mathbf{Z}$ & & & \\
\hline BMl & Corpus callosum & 14 & -32 & 30 & -2.390 & 0.000045657 & 58 \\
\hline
\end{tabular}

BMI, body mass index; FA, fractional anisotropy; MNI, Montreal Neurological Institute; SDM, seed-based d mapping; T2DM, type 2 diabetes.

which pass through the temporal lobe, such as IFOF and $\operatorname{ILF}(33,36$, 54). Besides, some fibers of IFOF and UF are located in the external capsule, which associates the hippocampus and amygdala with prefrontal and orbitofrontal cortices $(54,65)$. Previous studies already indicated that atrophy in temporal lobe, hippocampus, and orbitofrontal regions occurred in $\operatorname{T2DM}(2,13,54)$, and also evidence has shown that atrophy in these areas is one of the earliest neuroanatomical changes in Alzheimer's dementia (2, 36, 53). 
Among the eight studies included in our meta-analysis, four of them reported microstructural abnormalities in temporal regions. Given that the vital role that the temporal lobe, the hippocampus, and the orbitofrontal cortex play in cognitive processes such as learning, memory, and decision making $(66,67)$, we conjectured that disruptions of WM in IFOF, ILF, and UF might be related with cognitive function deficits in T2DM patients. Besides, the comorbidity of depression and T2DM is quite common (1821 ), and disrupted WM connectivity in inferior network has also been constantly found in MDD patients $(29,68,69)$. Thus, microarchitecture alterations in the inferior network might also underlie potential affective changes in T2DM.

The CC is the largest interhemispheric WM commissure connecting the cerebral hemispheres, and plays crucial role in interhemispheric communication and cognitive processes (70). Microstructural changes in this core WM tract were found not only in T2DM patients in numerous research $(34-36,38,54)$, but also in patients with cognitive impairment $(38,71,72)$ and patients with $\operatorname{MDD}(29,68,73,74)$. Therefore, decreased FA in the CC observed in our meta-analysis may underlie the deficits in cognitive processing and emotional modulation in patients with T2DM. Besides, there was a negative correlation between FA in the CC and BMI in T2DM patients revealed by meta-regression analysis. This was consistent with previous findings that higher BMI was associated with FA reductions in the CC in healthy cohorts $(75,76)$. There were DTI studies on BMI-related WM abnormalities suggesting a primordial effect of BMI on brain circuits involved in reward processing and emotion regulation (77), or even on the entire brain (75). Furthermore, there was evidence that alterations in white matter were associated with several obesity-related conditions such as cardiovascular risk factors including metabolic syndrome (78). Therefore, our finding might suggest disrupted CC microstructures as an BMI-related neurobiological marker of T2DM. The other WM tracts showed non-significant regression results, probably due to a relatively strict $P$-value in the process of statistics. Neurologic changes in the left inferior network and the left olfactory cortex might also be associated with metabolic syndrome related symptoms and these regions should receive full considerations.

It is particularly noteworthy that the left olfactory cortex exhibited decreased FA in T2DM patients. Current evidence implied that olfactory function is associated with the emergence of prodromal $\operatorname{AD}(79,80)$. Scholars assumed that olfactory impairments might reflect the onset of $\mathrm{AD}$, amnestic mild cognitive impairment (MCI), and the presence of amyloid- $\beta(\mathrm{A} \beta)$ and tau pathology $(79,81-86)$. Thus, FA reductions in the left olfactory cortex might be served as an early prediction of cognitive impairment in T2DM patients. This was of great significance for early detection of potential cognitive decline and dementia in T2DM patients. Moreover, olfactory function was also found to be related to the pathogenesis of MDD (87). Olfactory sulcus structural abnormality might be a trait-related marker of vulnerability to MDD (88). In consideration of the high prevalence of comorbidity of depression and T2DM, olfactory cortex alterations might be involved in the pathophysiology of the co-morbidity.

Several limitations of this study should be noted. Firstly, as the number of studies included in our meta-analysis was small, we were not able to perform separate subgroup meta-analyses for clinical variables such as cognition status, depression severity, and BMI, or methodological differences such as VBA and TBSS, which would likely diversify the results. Secondly, the data acquisition parameters, participants characteristics and clinical variables in the included studies were heterogeneous. It is not possible to eliminate these differences by statistical means. Thirdly, our analysis was limited to WM diffusion changes thereby not including the large amount of research on GM volume or WM volume. Future meta-analysis could include VBM studies for a more comprehensive perspective of the brain microarchitecture. Last but not least, it is meaningful to work on the reversibility of nerve damage, but the present metaanalysis and the literatures included in our research are all crosssectional design. Longitudinal studies with respect to reversibility of the neurodegeneration of T2DM is of great importance and should be addressed in the future.

\section{CONCLUSION}

The present meta-analysis indicated that T2DM patients demonstrated significant FA reductions in the left inferior network, the CC and the left olfactory cortex. Among them, FA of the CC had a negative correlation with BMI in the patients group. These findings supported the opinion that T2DM could lead to subtle WM structural alterations, which might be associated with cognitive deficits or emotional distress in T2DM patients. This helps us better understand the neural mechanism underlying neurodegeneration in T2DM.

\section{DATA AVAILABILITY STATEMENT}

The original contributions presented in the study are included in the article/supplementary material. Further inquiries can be directed to the corresponding author.

\section{AUTHOR CONTRIBUTIONS}

$\mathrm{CZ}$ designed the study and revised the manuscript. CZ wrote the initial manuscript. CZ and JL collected the data and undertook the statistical analysis. MD and LP assisted with data collection and statistical analysis and modified the paper. HL, YW, SW, SG, and GY assisted with data collection and data analysis. YC and XX critically reviewed and modified the paper. All authors contributed to the article and approved the submitted version.

\section{FUNDING}

This study was supported by the Medical and Health Science and Technology Development Plan of Shandong Province (202003061210), and the Supporting Fund for Teachers' Research of Jining Medical University. The Supporting Fund for Teachers' Research of Jining Medical University (600903001). 


\section{REFERENCES}

1. Krentz N, Gloyn A. Insights Into Pancreatic Islet Cell Dysfunction From Type 2 Diabetes Mellitus Genetics. Nat Rev Endocrinol (2020) 16(4):202-12. doi: 10.1038/s41574-020-0325-0

2. Brundel M, Kappelle LJ, Biessels GJ. Brain Imaging in Type 2 Diabetes. Eur Neuropsychopharmacol (2014) 24(12):1967-81. doi: 10.1016/ j.euroneuro.2014.01.023

3. Li Y, Teng D, Shi X, Qin G, Qin Y, Quan H, et al. Prevalence of Diabetes Recorded in Mainland China Using 2018 Diagnostic Criteria From the American Diabetes Association: National Cross Sectional Study. Bmj (2020) 369. doi: $10.1136 /$ bmj.m997

4. Klein JP, Waxman SG. The Brain in Diabetes: Molecular Changes in Neurons and Their Implications for End-Organ Damage. Lancet Neurol (2003) 2 (9):548-54. doi: 10.1016/s1474-4422(03)00503-9

5. Biessels GJ, Strachan MWJ, Visseren FLJ, Kappelle LJ, Whitmer RA. Dementia and Cognitive Decline in Type 2 Diabetes and Prediabetic Stages: Towards Targeted Interventions. Lancet Diabetes Endocrinol (2014) 2 (3):246-55. doi: 10.1016/s2213-8587(13)70088-3

6. Ryan JP, Fine DF, Rosano C. Type 2 Diabetes and Cognitive Impairment: Contributions From Neuroimaging. J Geriatr Psychiatry Neurol (2014) 27 (1):47-55. doi: 10.1177/0891988713516543

7. Biessels GJ, Nobili F, Teunissen CE, Simó R, Scheltens P. Understanding Multifactorial Brain Changes in Type 2 Diabetes: A Biomarker Perspective. Lancet Neurol (2020) 19(8):699-710. doi: 10.1016/s1474-4422(20)30139-3

8. Lu FP, Lin KP, Kuo HK. Diabetes and the Risk of Multi-System Aging Phenotypes: A Systematic Review and Meta-Analysis. PloS One (2009) 4(1): e4144. doi: 10.1371/journal.pone.0004144

9. Danna SM, Graham E, Burns RJ, Deschenes SS, Schmitz N. Association Between Depressive Symptoms and Cognitive Function in Persons With Diabetes Mellitus: A Systematic Review. PloS One (2016) 11(8):e0160809. doi: 10.1371/journal.pone. 0160809

10. Moran C, Beare R, Wang W, Callisaya M, Srikanth V. Alzheimer's Disease Neuroimaging I. Type 2 Diabetes Mellitus, Brain Atrophy, and Cognitive Decline. Neurology (2019) 92(8):e823-e30. doi: 10.1212/WNL. 0000000000006955

11. Callisaya ML, Beare R, Moran C, Phan T, Wang W, Srikanth VK. Type 2 Diabetes Mellitus, Brain Atrophy and Cognitive Decline in Older People: A Longitudinal Study. Diabetologia (2019) 62(3):448-58. doi: 10.1007/s00125018-4778-9

12. Shi L, Cheng Y, Xu Y, Shen Z, Lu Y, Zhou C, et al. Effects of Hypertension on Cerebral Cortical Thickness Alterations in Patients With Type 2 Diabetes. Diabetes Res Clin Pract (2019) 157:107872. doi: 10.1016/j.diabres.2019.107872

13. Rosenberg J, Lechea N, Pentang GN, Shah NJ. What Magnetic Resonance Imaging Reveals - A Systematic Review of the Relationship Between Type II Diabetes and Associated Brain Distortions of Structure and Cognitive Functioning. Front Neuroendocrinol (2019) 52:79-112. doi: 10.1016/ j.yfrne.2018.10.001

14. Brundel M, van den Heuvel M, de Bresser J, Kappelle LJ, Biessels GJ. Utrecht Diabetic Encephalopathy Study G. Cerebral Cortical Thickness in Patients With Type 2 Diabetes. J Neurol Sci (2010) 299(1-2):126-30. doi: 10.1016/ j.jns.2010.08.048

15. Yau PL, Javier DC, Ryan CM, Tsui WH, Ardekani BA, Ten S, et al. Preliminary Evidence for Brain Complications in Obese Adolescents With Type 2 Diabetes Mellitus. Diabetologia (2010) 53(11):2298-306. doi: 10.1007/ s00125-010-1857-y

16. Pouwer F. Should We Screen for Emotional Distress in Type 2 Diabetes Mellitus? Nat Rev Endocrinol (2009) 5(12):665-71. doi: 10.1038/ nrendo.2009.214

17. Xia W, Luo Y, Chen YC, Zhang D, Bo F, Zhou P, et al. Disrupted Functional Connectivity of the Amygdala is Associated With Depressive Mood in Type 2 Diabetes Patients. J Affect Disord (2018) 228:207-15. doi: 10.1016/ j.jad.2017.12.012

18. Darwish L, Beroncal E, Sison MV, Swardfager W. Depression in People With Type 2 Diabetes: Current Perspectives. Diabetes Metab Syndr Obes (2018) 11:333-43. doi: 10.2147/DMSO.S106797

19. Huang CJ, Hsieh HM, Tu HP, Jiang HJ, Wang PW, Lin CH. Major Depressive Disorder in Patients With Type 2 Diabetes Mellitus: Prevalence and Clinical
Characteristics. J Affect Disord (2018) 227:141-8. doi: 10.1016/j.jad. 2017.09.044

20. Hussain S, Habib A, Singh A, Akhtar M, Najmi AK. Prevalence of Depression Among Type 2 Diabetes Mellitus Patients in India: A Meta-Analysis. Psychiatry Res (2018) 270:264-73. doi: 10.1016/j.psychres.2018.09.037

21. Salinero-Fort MA, Gomez-Campelo P, San Andres-Rebollo FJ, CardenasValladolid J, Abanades-Herranz JC, Carrillo de Santa Pau E, et al. Prevalence of Depression in Patients With Type 2 Diabetes Mellitus in Spain (the DIADEMA Study): Results From the MADIABETES Cohort. BMJ Open (2018) 8(9):e020768. doi: 10.1136/bmjopen-2017-020768

22. van Sloten TT, Sedaghat S, Carnethon MR, Launer LJ, Stehouwer CDA. Cerebral Microvascular Complications of Type 2 Diabetes: Stroke, Cognitive Dysfunction, and Depression. Lancet Diabetes Endocrinol (2020) 8(4):325-36. doi: 10.1016/s2213-8587(19)30405-x

23. Le Bihan D, Mangin JF, Poupon C, Clark CA, Pappata S, Molko N, et al. Diffusion Tensor Imaging: Concepts and Applications. J Magnet Resonance Imaging: JMRI (2001) 13(4):534-46. doi: 10.1002/jmri.1076

24. Beaulieu C. The Basis of Anisotropic Water Diffusion in the Nervous System a Technical Review. NMR Biomed (2002) 15(7-8):435-55. doi: 10.1002/ nbm.782

25. Schiavone F, Charlton RA, Barrick TR, Morris RG, Markus HS. Imaging AgeRelated Cognitive Decline: A Comparison of Diffusion Tensor and Magnetization Transfer MRI. J Magnet Resonance Imaging: JMRI (2009) 29 (1):23-30. doi: 10.1002/jmri.21572

26. Zhuang L, Sachdev PS, Trollor JN, Kochan NA, Reppermund S, Brodaty H, et al. Microstructural White Matter Changes in Cognitively Normal Individuals At Risk of Amnestic MCI. Neurology (2012) 79(8):748-54. doi: 10.1212/WNL.0b013e3182661f4d

27. Koch K, Reess TJ, Rus OG, Zimmer C, Zaudig M. Diffusion Tensor Imaging (DTI) Studies in Patients With Obsessive-Compulsive Disorder (OCD): A Review. J Psychiatr Res (2014) 54:26-35. doi: 10.1016/j.jpsychires.2014.03.006

28. Smith SM, Jenkinson M, Johansen-Berg H, Rueckert D, Nichols TE, Mackay CE, et al. Tract-Based Spatial Statistics: Voxelwise Analysis of Multi-Subject Diffusion Data. NeuroImage (2006) 31(4):1487-505. doi: 10.1016/ j.neuroimage.2006.02.024

29. Jiang J, Zhao YJ, Hu XY, Du MY, Chen ZQ, Wu M, et al. Microstructural Brain Abnormalities in Medication-Free Patients With Major Depressive Disorder: A Systematic Review and Meta-Analysis of Diffusion Tensor Imaging. J Psychiatry Neurosci: JPN (2017) 42(3):150-63. doi: 10.1503/jpn.150341

30. Yau PL, Javier D, Tsui W, Sweat V, Bruehl H, Borod JC, et al. Emotional and Neutral Declarative Memory Impairments and Associated White Matter Microstructural Abnormalities in Adults With Type 2 Diabetes. Psychiatry Res (2009) 174(3):223-30. doi: 10.1016/j.pscychresns.2009.04.016

31. Hsu JL, Chen YL, Leu JG, Jaw FS, Lee CH, Tsai YF, et al. Microstructural White Matter Abnormalities in Type 2 Diabetes Mellitus: A Diffusion Tensor Imaging Study. NeuroImage (2012) 59(2):1098-105. doi: 10.1016/ j.neuroimage.2011.09.041

32. Xiong Y, Zhang S, Shi J, Fan Y, Zhang Q, Zhu W. Application of Neurite Orientation Dispersion and Density Imaging to Characterize Brain Microstructural Abnormalities in Type-2 Diabetics With Mild Cognitive Impairment. J Magnet Resonance Imaging: JMRI (2019) 50(3):889-98. doi: $10.1002 /$ jmri.26687

33. Zhang JH, Xu HZ, Shen QF, Lin YZ, Sun CK, Sha L, et al. Nepsilon(Carboxymethyl)-Lysine, White Matter, and Cognitive Function in Diabetes Patients. Can J Neurol Sci Le J Canadien Des Sci Neurol (2016) 43(4):518-22. doi: $10.1017 /$ cjn.2015.398

34. Kim DJ, Yu JH, Shin MS, Shin YW, Kim MS. Hyperglycemia Reduces Efficiency of Brain Networks in Subjects With Type 2 Diabetes. PloS One (2016) 11(6):e0157268. doi: 10.1371/journal.pone.0157268

35. Nouwen A, Chambers A, Chechlacz M, Higgs S, Blissett J, Barrett TG, et al. Microstructural Abnormalities in White and Gray Matter in Obese Adolescents With and Without Type 2 Diabetes. NeuroImage Clin (2017) 16:43-51. doi: 10.1016/j.nicl.2017.07.004

36. Yoon S, Cho H, Kim J, Lee DW, Kim GH, Hong YS, et al. Brain Changes in Overweight/Obese and Normal-Weight Adults With Type 2 Diabetes Mellitus. Diabetologia (2017) 60(7):1207-17. doi: 10.1007/s00125-017-4266-7

37. Hoogenboom WS, Marder TJ, Flores VL, Huisman S, Eaton HP, Schneiderman JS, et al. Cerebral White Matter Integrity and Resting-State 
Functional Connectivity in Middle-Aged Patients With Type 2 Diabetes. Diabetes (2014) 63(2):728-38. doi: 10.2337/db13-1219

38. Zhang J, Wang Y, Wang J, Zhou X, Shu N, Wang Y, et al. White Matter Integrity Disruptions Associated With Cognitive Impairments in Type 2 Diabetic Patients. Diabetes (2014) 63(11):3596-605. doi: 10.2337/db14-0342

39. Fox PT, Lancaster JL, Laird AR, Eickhoff SB. Meta-Analysis in Human Neuroimaging: Computational Modeling of Large-Scale Databases. Annu Rev Neurosci (2014) 37:409-34. doi: 10.1146/annurev-neuro-062012-170320

40. Radua J, Mataix-Cols D, Phillips ML, El-Hage W, Kronhaus DM, Cardoner N, et al. A New Meta-Analytic Method for Neuroimaging Studies That Combines Reported Peak Coordinates and Statistical Parametric Maps. Eur Psychiatry: J Assoc Eur Psychiatrists (2012) 27(8):605-11. doi: 10.1016/j.eurpsy.2011.04.001

41. Turkeltaub PE, Eden GF, Jones KM, Zeffiro TA. Meta-Analysis of the Functional Neuroanatomy of Single-Word Reading: Method and Validation. NeuroImage (2002) 16(3-part-PA):765-80. doi: 10.1006/ nimg.2002.1131

42. Wager TD, Lindquist M, Kaplan L. Meta-Analysis of Functional Neuroimaging Data: Current and Future Directions. Soc Cogn Affect Neuroence (2007) 2(2):150. doi: 10.1093/scan/nsm015

43. Radua J, Mataix-Cols D. Voxel-Wise Meta-Analysis of Grey Matter Changes in Obsessive-Compulsive Disorder. Br J Psychiatry: J Ment Sci (2009) 195 (5):393-402. doi: 10.1192/bjp.bp.108.055046

44. Radua J, Rubia K, Canales-Rodriguez EJ, Pomarol-Clotet E, Fusar-Poli P, Mataix-Cols D. Anisotropic Kernels for Coordinate-Based Meta-Analyses of Neuroimaging Studies. Front Psychiatry (2014) 5:13. doi: 10.3389/ fpsyt.2014.00013

45. Suo X, Lei D, Li W, Li L, Dai J, Wang S, et al. Altered White Matter Microarchitecture in Parkinson's Disease: A Voxel-Based Meta-Analysis of Diffusion Tensor Imaging Studies. Front Med (2020) 15(1):125-38. doi: 10.1007/s11684-019-0725-5

46. Mihaescu AS, Masellis M, Graff-Guerrero A, Kim J, Criaud M, Cho SS, et al. Brain Degeneration in Parkinson's Disease Patients With Cognitive Decline: A Coordinate-Based Meta-Analysis. Brain Imaging Behav (2019) 13(4):102134. doi: 10.1007/s11682-018-9922-0

47. Nortje G, Stein DJ, Radua J, Mataix-Cols D, Horn N. Systematic Review and Voxel-Based Meta-Analysis of Diffusion Tensor Imaging Studies in Bipolar Disorder. J Affect Disord (2013) 150(2):192-200. doi: 10.1016/j.jad. 2013.05.034

48. Hu X, Zhang L, Bu X, Li H, Gao Y, Lu L, et al. White Matter Disruption in Obsessive-Compulsive Disorder Revealed by Meta-Analysis of Tract-Based Spatial Statistics. Depress Anxiety (2020) 37(7):620-31. doi: 10.1002/da.23008

49. Zhang Z, Ping L, Zhai A, Zhou C. Microstructural White Matter Abnormalities in Obsessive-Compulsive Disorder: A Coordinate-Based Meta-Analysis of Diffusion Tensor Imaging Studies. Asian J Psychiatry (2020) 55. doi: 10.1016/j.ajp.2020.102467

50. Radua J, Via E, Catani M, Mataix-Cols D. Voxel-Based Meta-Analysis of Regional White-Matter Volume Differences in Autism Spectrum Disorder Versus Healthy Controls. psychol Med (2011) 41(7):1539-50. doi: 10.1017/ S0033291710002187

51. Liu J, Fan W, Jia Y, Su X, Wu W, Long X, et al. Altered Gray Matter Volume in Patients With Type 1 Diabetes Mellitus. Front Endocrinol (2020) 11:45. doi: $10.3389 /$ fendo.2020.00045

52. Liu J, Liu T, Wang W, Ma L, Ma X, Shi S, et al. Reduced Gray Matter Volume in Patients With Type 2 Diabetes Mellitus. Front Aging Neurosci (2017) 9:161. doi: $10.3389 /$ fnagi.2017.00161

53. Wu G, Lin L, Zhang Q, Wu J. Brain Gray Matter Changes in Type 2 Diabetes Mellitus: A Meta-Analysis of Whole-Brain Voxel-Based Morphometry Study. J Diabetes its Complications (2017) 31(12):1698-703. doi: 10.1016/ j.jdiacomp.2017.09.001

54. Sanjari Moghaddam H, Ghazi Sherbaf F, Aarabi MH. Brain Microstructural Abnormalities in Type 2 Diabetes Mellitus: A Systematic Review of Diffusion Tensor Imaging Studies. Front Neuroendocrinol (2019) 55:100782. doi: 10.1016/j.yfrne.2019.100782

55. Moher D, Liberati A, Tetzlaff J, Altman DGGroup P. Preferred Reporting Items for Systematic Reviews and Meta-Analyses: The PRISMA Statement. J Clin Epidemiol (2009) 62(10):1006-12. doi: 10.1016/j.jclinepi.2009.06.005

56. Liberati A, Altman DG, Tetzlaff J, Mulrow C, Gotzsche PC, Ioannidis JP, et al. The PRISMA Statement for Reporting Systematic Reviews and Meta-Analyses of Studies That Evaluate Healthcare Interventions: Explanation and Elaboration. Bmj (2009) 339:b2700. doi: 10.1136/bmj.b2700

57. Moher D, Liberati A, Tetzlaff J, Altman DG. Preferred Reporting Items for Systematic Reviews and Meta-Analyses: The PRISMA Statement. PloS Med (2009) 62(10):1006-12. doi: 10.1371/journal.pmed.1000097

58. Muller VI, Cieslik EC, Laird AR, Fox PT, Radua J, Mataix-Cols D, et al. Ten Simple Rules for Neuroimaging Meta-Analysis. Neurosci Biobehav Rev (2018) 84:151-61. doi: 10.1016/j.neubiorev.2017.11.012

59. Albajes-Eizagirre A, Solanes A, Vieta E, Radua J. Voxel-Based Meta-Analysis Via Permutation of Subject Images (PSI): Theory and Implementation for SDM. NeuroImage (2019) 186:174-84. doi: 10.1016/j.neuroimage.2018.10.077

60. Yang C, Hu X, Luo Q, Kuang W, Lui S, Huang X, et al. Psychoradiologic Abnormalities of White Matter in Patients With Bipolar Disorder: Diffusion Tensor Imaging Studies Using Tract-Based Spatial Statistics. J Psychiatry Neurosci (2019) 44(1):32-44. doi: 10.1503/jpn.170221

61. van Bloemendaal L, Ijzerman RG, Ten Kulve JS, Barkhof F, Diamant M, Veltman DJ, et al. Alterations in White Matter Volume and Integrity in Obesity and Type 2 Diabetes. Metab Brain Dis (2016) 31(3):621-9. doi: 10.1007/s11011-016-9792-3

62. Liang Y, Zhang H, Tan X, Liu J, Qin C, Zeng H, et al. Local Diffusion Homogeneity Provides Supplementary Information in T2DM-Related Wm Microstructural Abnormality Detection. Front Neurosci (2019) 13:63. doi: $10.3389 /$ fnins. 2019.00063

63. Alger JR. The Diffusion Tensor Imaging Toolbox. J Neurosci (2012) 32 (22):7418-28. doi: 10.1523/JNEUROSCI.4687-11.2012

64. Tomlinson DR, Gardiner NJ. Glucose Neurotoxicity. Nat Rev Neurosci (2008) 9(1):36-45. doi: 10.1038/nrn2294

65. Shott ME, Cornier MA, Mittal VA, Pryor TL, Orr JM, Brown MS, et al. Orbitofrontal Cortex Volume and Brain Reward Response in Obesity. Int $J$ Obes (2015) 39(2):214-21. doi: 10.1038/ijo.2014.121

66. Bell B, Lin JJ, Seidenberg M, Hermann B. The Neurobiology of Cognitive Disorders in Temporal Lobe Epilepsy. Nat Rev Neurol (2011) 7(3):154-64. doi: $10.1038 /$ nrneurol.2011.3

67. Wikenheiser AM, Schoenbaum G. Over the River, Through the Woods: Cognitive Maps in the Hippocampus and Orbitofrontal Cortex. Nat Rev Neurosci (2016) 17(8):513-23. doi: 10.1038/nrn.2016.56

68. Liao Y, Huang X, Wu Q, Yang C, Kuang W, Du M, et al. Is Depression a Disconnection Syndrome? Meta-analysis of Diffusion Tensor Imaging Studies in Patients With MDD. J Psychiatry Neurosci JPN (2013) 38(1):49-56. doi: $10.1503 /$ jpn. 110180

69. Chen G, Guo Y, Zhu H, Kuang W, Bi F, Ai H, et al. Intrinsic Disruption of White Matter Microarchitecture in First-Episode, Drug-Naive Major Depressive Disorder: A Voxel-Based Meta-Analysis of Diffusion Tensor Imaging. Prog Neuropsychopharmacol Biol Psychiatry (2017) 76:179-87. doi: 10.1016/j.pnpbp.2017.03.011

70. Saito Y, Nobuhara K, Okugawa G, Takase K, Sugimoto T, Horiuchi M, et al. Corpus Callosum in Patients With Obsessive-Compulsive Disorder: Diffusion-Tensor Imaging Study. Radiology (2008) 246(2):536-42. doi: 10.1148/radiol.2462061469

71. Brueggen K, Dyrba M, Cardenas-Blanco A, Schneider A, Fliessbach K, Buerger K, et al. Structural Integrity in Subjective Cognitive Decline, Mild Cognitive Impairment and Alzheimer's Disease Based on Multicenter Diffusion Tensor Imaging. J Neurol (2019) 266(10):2465-74. doi: 10.1007/ s00415-019-09429-3

72. Lo Buono V, Palmeri R, Corallo F, Allone C, Pria D, Bramanti P, et al. Diffusion Tensor Imaging of White Matter Degeneration in Early Stage of Alzheimer's Disease: A Review. Int J Neurosci (2020) 130(3):243-50. doi: 10.1080/00207454.2019.1667798

73. Xu K, Jiang $\mathrm{W}$, Ren L, Ouyang $\mathrm{X}$, Jiang $\mathrm{Y}, \mathrm{Wu} \mathrm{F}$, et al. Impaired Interhemispheric Connectivity in Medication-Naive Patients With Major Depressive Disorder. J Psychiatry Neurosci: JPN (2013) 38(1):43-8. doi: $10.1503 /$ jpn.110132

74. Wise T, Radua J, Nortje G, Cleare A, Young A, Arnone D. Voxel-Based MetaAnalytical Evidence of Structural Disconnectivity in Major Depression and Bipolar Disorder. Biol Psychiatry (2016) 79(4):293-302. doi: 10.1016/ j.biopsych.2015.03.004

75. Repple J, Opel N, Meinert S, Redlich R, Hahn T, Winter NR, et al. Elevated Body-Mass Index is Associated With Reduced White Matter Integrity in Two 
Large Independent Cohorts. Psychoneuroendocrinology (2018) 91:179-85. doi: 10.1016/j.psyneuen.2018.03.007

76. Xu J, Li Y, Lin H, Sinha R, Potenza MN. Body Mass Index Correlates Negatively With White Matter Integrity in the Fornix and Corpus Callosum: A Diffusion Tensor Imaging Study. Hum Brain Mapp (2013) 34 (5):1044-52. doi: 10.1002/hbm.21491

77. Papageorgiou I, Astrakas LG, Xydis V, Alexiou GA, Bargiotas P, Tzarouchi L, et al. Abnormalities of Brain Neural Circuits Related to Obesity: A Diffusion Tensor Imaging Study. Magnet Resonance Imaging (2017) 37:116-21. doi: 10.1016/j.mri.2016.11.018

78. Alfaro FJ, Gavrieli A, Saade-Lemus P, Lioutas VA, Upadhyay J, Novak V. White Matter Microstructure and Cognitive Decline in Metabolic Syndrome: A Review of Diffusion Tensor Imaging. Metabol: Clin Exp (2018) 78:52-68. doi: 10.1016/j.metabol.2017.08.009

79. Murphy C. Olfactory and Other Sensory Impairments in Alzheimer Disease. Nat Rev Neurol (2019) 15(1):11-24. doi: 10.1038/s41582-018-0097-5

80. Swan GE, Carmelli D. Impaired Olfaction Predicts Cognitive Decline in Nondemented Older Adults. Neuroepidemiology (2002) 21(2):58-67. doi: $10.1159 / 000048618$

81. Dhilla Albers A, Asafu-Adjei J, Delaney MK, Kelly KE, Gomez-Isla T, Blacker D, et al. Episodic Memory of Odors Stratifies Alzheimer Biomarkers in Normal Elderly. Ann Neurol (2016) 80(6):846-57. doi: 10.1002/ana.24792

82. Vassilaki M, Christianson TJ, Mielke MM, Geda YE, Kremers WK, Machulda MM, et al. Neuroimaging Biomarkers and Impaired Olfaction in Cognitively Normal Individuals. Ann Neurol (2017) 81(6):871-82. doi: 10.1002/ana.24960

83. Murphy C, Jernigan TL, Fennema-Notestine C. Left Hippocampal Volume Loss in Alzheimer's Disease is Reflected in Performance on Odor Identification: A Structural MRI Study. J Int Neuropsychol Soc (2003) 9 (3):459-71. doi: 10.1017/S1355617703930116
84. Growdon ME, Schultz AP, Dagley AS, Amariglio RE, Hedden T, Rentz DM, et al. Odor Identification and Alzheimer Disease Biomarkers in Clinically Normal Elderly. Neurology (2015) 84(21):2153. doi: 10.1212/WNL. 0000000000001614

85. Lafaille-Magnan M-E, Poirier J, Etienne P, Tremblay-Mercier J, Frenette J, RosaNeto P, et al. Odor Identification as a Biomarker of Preclinical AD in Older Adults At Risk. Neurology (2017) 89(4):327-35. doi: 10.1212/WNL.0000000000004159

86. Attems J, Walker L, Jellinger KA. Olfactory Bulb Involvement in Neurodegenerative Diseases. Acta Neuropathol (2014) 127(4):459-75. doi: 10.1007/s00401-014-1261-7

87. Wang F, Wu X, Gao J, Li Y, Zhu Y, Fang Y. The Relationship of Olfactory Function and Clinical Traits in Major Depressive Disorder. Behav Brain Res (2020) 386:112594. doi: 10.1016/j.bbr.2020.112594

88. Takahashi T, Nishikawa Y, Yucel M, Whittle S, Lorenzetti V, Walterfang M, et al. Olfactory Sulcus Morphology in Patients With Current and Past Major Depression. Psychiatry Res Neuroimaging (2016) 255:60-5. doi: 10.1016/ j.pscychresns.2016.07.008

Conflict of Interest: The authors declare that the research was conducted in the absence of any commercial or financial relationships that could be construed as a potential conflict of interest.

Copyright $\odot 2021$ Zhou, Li, Dong, Ping, Lin, Wang, Wang, Gao, Yu, Cheng and Xu. This is an open-access article distributed under the terms of the Creative Commons Attribution License (CC BY). The use, distribution or reproduction in other forums is permitted, provided the original author(s) and the copyright owner(s) are credited and that the original publication in this journal is cited, in accordance with accepted academic practice. No use, distribution or reproduction is permitted which does not comply with these terms. 